Revue des patrimoines

19 | 2012

Patrimoines et conservation préventive. Pratiques comparées et nouveaux enjeux

\title{
L'intégration de la conservation préventive dans les phases de la programmation architecturale
}

\section{Stéphanie Kuhn}

\section{(2) OpenEdition}

Journals

Édition électronique

URL : http://journals.openedition.org/insitu/9980

DOI : $10.4000 /$ insitu.9980

ISSN : 1630-7305

Éditeur

Ministère de la culture

Référence électronique

Stéphanie Kuhn, «L'intégration de la conservation préventive dans les phases de la programmation architecturale», In Situ [En ligne], 19 | 2012, mis en ligne le 03 janvier 2013, consulté le 01 mai 2019 URL : http://journals.openedition.org/insitu/9980 ; DOI : 10.4000/insitu.9980

Ce document a été généré automatiquement le 1 mai 2019.

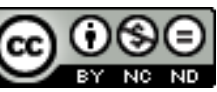

In Situ Revues des patrimoines est mis à disposition selon les termes de la licence Creative Commons Attribution - Pas d'Utilisation Commerciale - Pas de Modification 4.0 International. 


\title{
L'intégration de la conservation préventive dans les phases de la programmation architecturale
}

\author{
Stéphanie Kuhn
}

\section{Préambule}

1 Tout projet de création ou de rénovation de musée obéit à un objectif louable, celui d'améliorer les conditions anciennes d'exploitation, de conservation et de diffusion du patrimoine ${ }^{1}$. Il serait tout à fait malhonnête intellectuellement de remettre en cause ces objectifs et les résultats globalement obtenus. En effet, les projets récents constituent une amélioration sensible des conditions précédentes dans lesquelles les équipements étaient exploités. Néanmoins, si les objectifs sont globalement atteints, n'est-on pas en droit, au regard des sommes publiques dépensées et des énergies mobilisées, d'atteindre un niveau optimal faisant référence, ou tout du moins d'obtenir des musées qu'ils soient exemplaires en termes d'exploitation et de conservation des collections, sans renier une composante de leurs missions (la valorisation et la diffusion) ${ }^{2}$ au profit d'une autre ? Car le musée comme tout site patrimonial n'est-il pas le révélateur de la philosophie même de la conservation préventive puisqu'il est le lieu qui gère le nécessaire compromis entre la conservation des biens culturels dont il a la charge et leur diffusion. Il s'inscrit à la fois dans une démarche de long terme et dans une démarche de court terme visant la diffusion immédiate du savoir (la consommation du patrimoine) vers un plus large public possible. 


\section{Contexte de réalisation de l'étude et de la réflexion}

2 Ce sujet est un des éléments abordés dans le cadre du mémoire parachevant l'année universitaire du Master Pro de conservation préventive à l'université Paris I sous la direction de Denis Guillemard en 2009/2010.

\section{Approche globale de l'étude réalisée}

Le travail ${ }^{3}$ effectué dans le cadre du Master de Conservation Préventive visait à mettre en perspective et à problématiser l'intégration de la conservation préventive dans le processus d'un projet partant de la rédaction du PSC jusqu'à la livraison du musée. Ce processus se déroule sur de longues années, jalonnées de validations, épreuves, compromis, chaque projet ayant sa propre Histoire et «ses histoires». Malgré la singularité propre à chaque projet, les objectifs du mémoire de master concerné, étaient d'identifier grâce à un panel de musées analysés : (fig. ${ }^{\circ} \mathbf{1}$ )

- les schémas d'intégration de la conservation préventive et de ses spécialistes au cours de la réalisation des projets;

- la façon dont les objectifs de conservation préventive étaient affirmés puis intégrés comme paramètre opérationnel et outil d'aide à la décision au même titre que les paramètres économiques et techniques ;

- les problèmes rencontrés et leurs occurrences dans plusieurs études de cas.

Figure 1

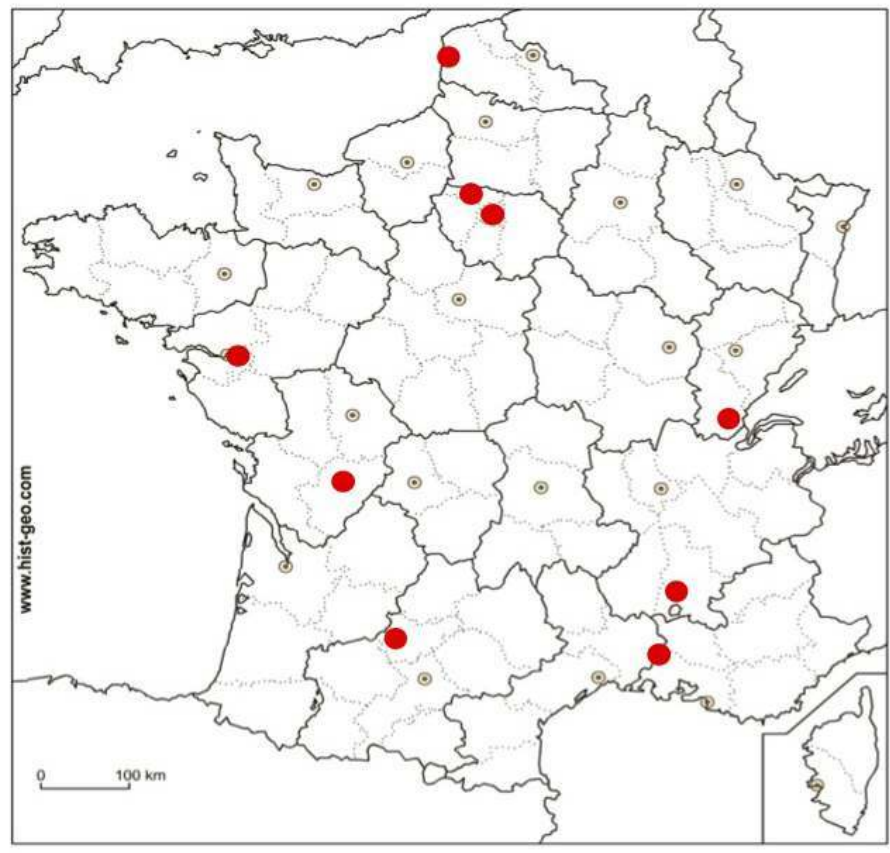

Figure 1: cartographie des cas analysés

Cartographie des cas analysés, 2009-2010.

(c) Stéphanie Kuhn-Likes. 


\section{Pourquoi questionner le rôle de la conservation préventive dans la phase de programmation?}

4 Les résultats obtenus par les évaluations ont permis de mettre en lumière et/ou de confirmer plusieurs problématiques récurrentes dont :

- la difficulté de maintenir les objectifs qualitatifs induit par la conservation préventive au moment des choix stratégiques faisant intervenir les facteurs temps ou coût (deux «épées de Damoclès)"

- le rôle déterminant des études de programmation destinées à faire basculer un projet en phase opérationnelle.

5 Au regard de ces enjeux, il apparait plus qu'opportun de s'interroger sur la prise en compte de la conservation préventive dans cette phase en particulier.

\section{Enjeux de la conservation préventive dans les études de programmation}

\section{La phase de programmation, un moment charnière du projet}

6 Notre objectif n'est pas ici de présenter la programmation architecturale en tant que telle. Nous renvoyons le lecteur aux textes publiés par la MICQP ${ }^{4}$ :

7 «Outil fondamental de la maitrise de la qualité du projet, la phase de programmation permet au Maître d'ouvrage de définir sa commande (en fonction de ses objectifs et de ses moyens) et de la maîtriser tout au long du processus de réalisation opérationnelle, pour aboutir à un projet satisfaisant tant qualitativement que techniquement.

La programmation vise à :

- clarifier, définir et préciser sa commande ;

- mettre en cohérence les objectifs initiaux d'un projet et sa conception, sa réalisation et sa gestion ;

- s'organiser autour du projet. $»^{5}$

(fig. $n^{\circ} 2$ ) 


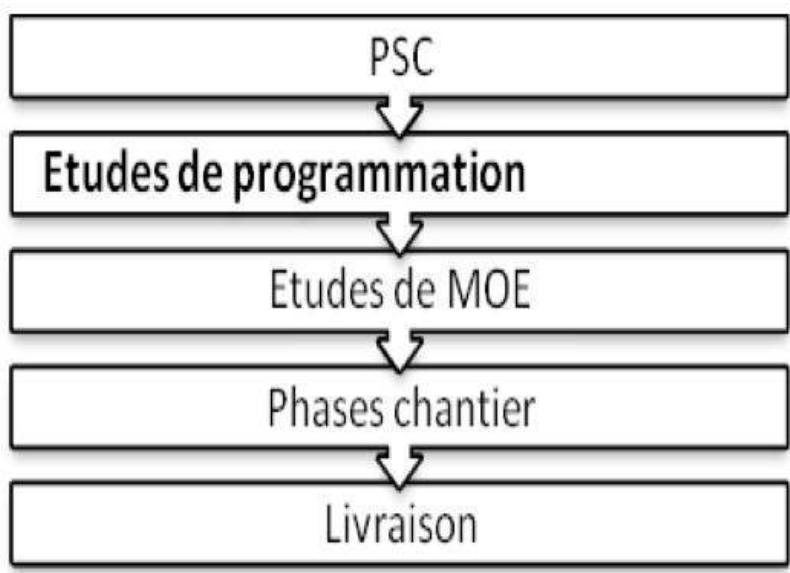

\section{Figure 2: Schéma de déroulement des Étapes d'u projet}

Schéma de déroulement des étapes du projet, 2009-2010.

(c) Stéphanie Kuhn-Likes. création d'un bâtiment neuf ou de la restructuration d'un bâtiment préexistant, la phase de programmation constitue un moment crucial pour l'articulation entre le travail scientifique et le travail des maîtres d'œuvre.

Elle concentre des étapes structurantes telles les phases :

- d'analyse,

- d'études de la faisabilité des projets tant en termes conceptuels, architectural, technique qu'économique

avant d'aboutir à la rédaction du programme qui sera la traduction des intentions du Maitre d'ouvrage et les termes de sa commande au service des futurs maîtres d'œuvre qui auront la charge de la réalisation quantitativement et qualitativement. culturels d'un établissement et un faisceau de données spatiales, physiques, économiques, humaines, institutionnelles, réglementaires... Elles déterminent, ainsi les fondements qui font basculer un projet en phase opérationnelle. 


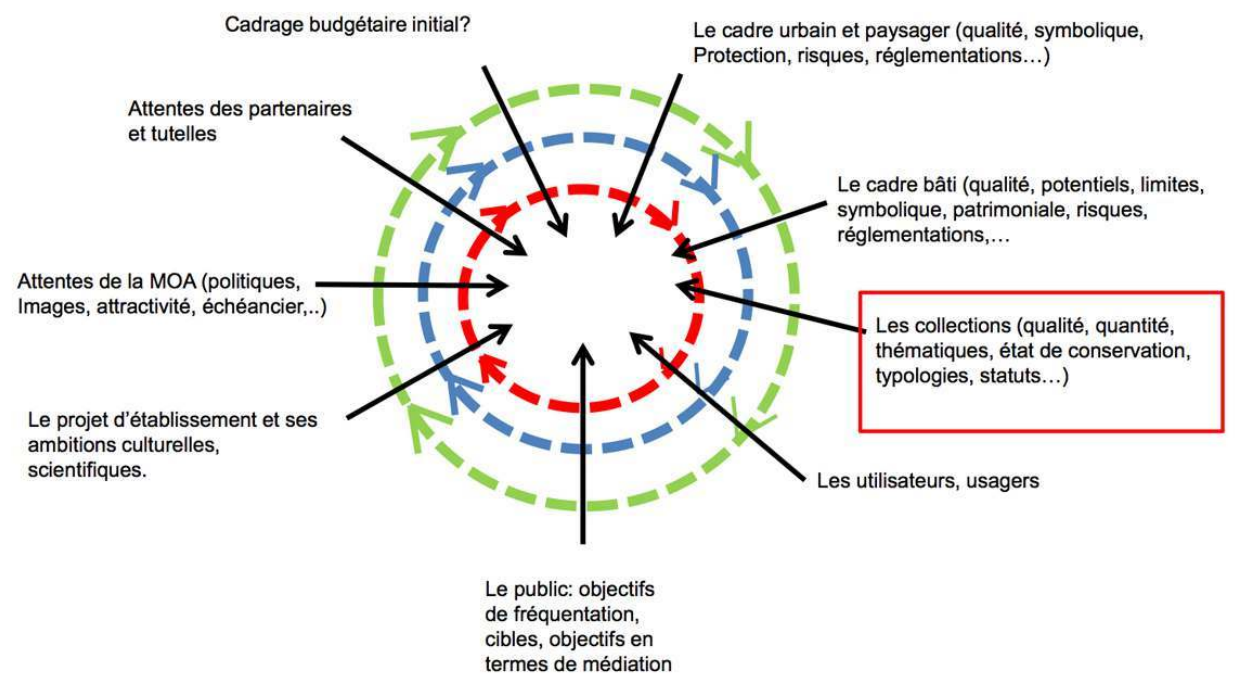

Figure 3: les différents paramètres

Les différents paramètres, 2009-2010.

(c) Stéphanie Kuhn-Likes.

La rigueur méthodologique des études de programmation est donc essentielle pour aider les maîtres d'ouvrage à définir l'envergure et le niveau qualitatif de leur projet muséographique et d'en assurer la faisabilité et la maîtrise sur le long terme.

Les différentes échelles du projet doivent être mises en perspective :

- L'échelle du site et des bâtiments concernés : il s'agit pour chaque projet de considérer l'ensemble des conditions physiques offertes par les espaces des bâtiments du site tant d'un point de vue architectural, patrimonial que d'un point de vue fonctionnel, technique et paysager/urbain.

- L'échelle muséologique qui intègre toutes les données liées au projet culturel et scientifique du musée y compris la connaissance des collections (qualitativement et quantitativement). Il s'agit notamment d'analyser la capacité des collections à répondre aux objectifs culturels et scientifiques, de prévoir les impacts programmatiques de la présentation (typologie, volumes, niveau d'éclairement, mode de présentation...), ...

La difficulté de la programmation est justement de concilier ces échelles et paramètres afin d'éviter des dysfonctionnements futurs au sein de l'équipement:

- Un défaut de prise en compte des besoins pour le fonctionnement du musée pouvant induire une sous évaluation des besoins en logistique muséographique, une organisation inopérante au regard de la définition de l'équipe qui sera en charge du musée, des choix techniques (notamment sur la gestion du climat) qui ne seront pas maîtrisables par les équipes du musée...;

- Une méconnaissance des collections pouvant engendrer en phase d'études de conception des erreurs en termes de choix architecturaux et muséographiques ;

- Une méconnaissance de certaines données sur le site peut engendrer des estimations économiques moins précises. 
Tout cela n'est pas anodin, puisqu'en définitive, les éléments n'ayant pu être anticipés pendant les phases de programmation sont susceptibles de ressurgir a posteriori lors des études de maîtrise d'œuvre puis au moment de la mise en fonctionnement de l'outil musée avec des conséquences majoritairement négatives pour la conservation des œuvres.

Or, les études de cas témoignent de la réelle difficulté dans de nombreuses situations de pouvoir mener à bien ce dialogue et cette anticipation et ce pour plusieurs raisons :

- Une décomposition de certains projets avec la programmation architecturale et technique d'une part et la programmation muséographique détaillée d'autre part, les deux étant menées souvent sur des temporalités différentes ${ }^{6}$ et/ou par des intervenants différents ;

- Concernant les collections et le projet muséographique : une connaissance lacunaire des collections notamment des collections en réserve; une programmation scientifique du parcours pas ou pas assez aboutie au lancement des études de programmation; un niveau d'accessibilité aux collections pouvant être nul ou suffisamment réduit pour empêcher une évaluation fine des futurs besoins ;

- Concernant le site : nature des plans, et relevés parfois très lacunaires ; absence de référents techniques.

\section{Quelle place pour la conservation préventive et les préventistes dans les phases de programmation aujourd'hui?} est de constater, que la conservation préventive reste encore trop souvent envisagée comme une discipline liée aux collections stricto sensu, notamment par les hommes de l'art, les programmistes, et différents acteurs techniques ou administratifs intervenant auprès des musées, certains jouant un rôle essentiel pour le fonctionnement futur de l'équipement (BET ${ }^{7}$, par exemple). ineloppés, celles-ci influent-elles suffisamment sur les projets architecturaux et muséographiques? Comment cette discipline est-elle intégrée dans les phases clés et notamment dans la phase de programmation architecturale et muséographique? 


\section{Mise en perspective pour une meilleure intégration de la conservation préventive}

Comment faire évoluer la situation? Il apparaît primordial de reconsidérer la conservation préventive non comme une discipline autonome mais à comme un objectif et une approche transversale à partager entre différents métiers, chacun disposant d'un ensemble d'outils et de méthodes (conservateurs, restaurateurs, programmistes, ingénieurs, architectes, muséographes...).

Les différents problèmes constatés et précités nous amènent à considérer plusieurs pistes d'évolution dont certaines sont déjà à l'œuvre (de façon empirique) mais sans avoir totalement permis d'évaluer les incidences générées.

\section{La conduite de projet ${ }^{8}$ : au-delà de la programmation, des études préalables essentielles}

Dans le cas de la réalisation d'un projet architectural et muséographique, le PSC pourrait davantage jouer le rôle d'outil généralisant notamment l'intégration de la programmation scientifique des collections (et de fait une connaissance poussée des collections) $)^{9}$, ce qui ne semble pas encore généralisé.

(fig. $\left.n^{\circ} 4\right)\left(\right.$ fig. $\left.n^{\circ} 5\right)$

Figure 4

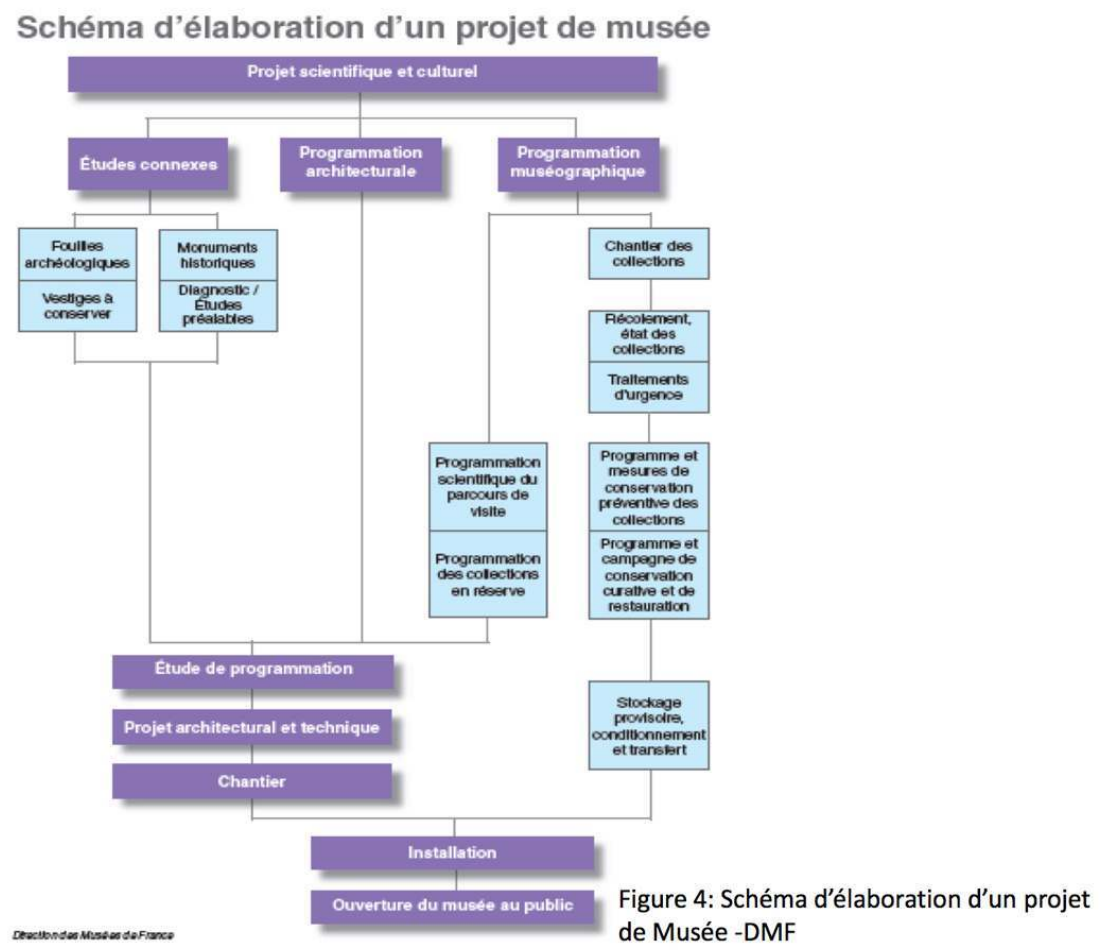

Schéma d'élaboration d'un projet de musée.

(c) Ministère de la Culture, SMF. 
Figure 5

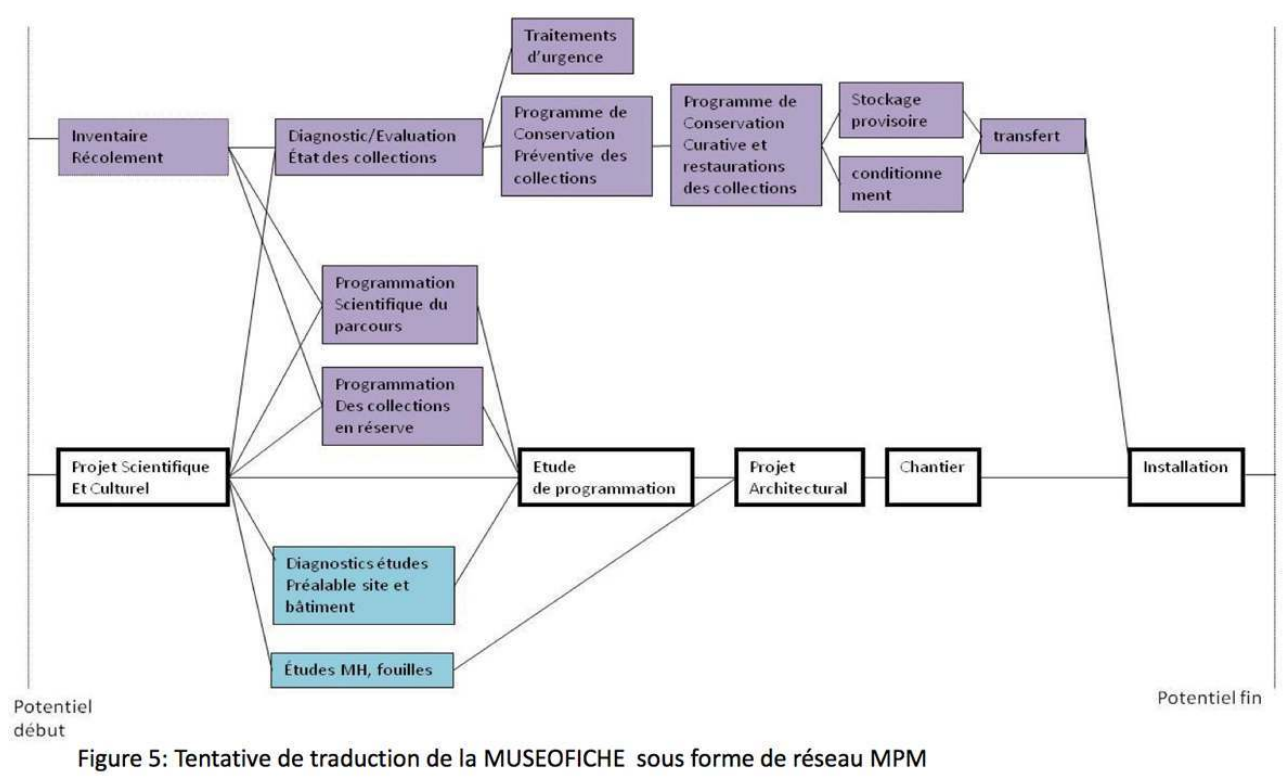

Tentative de traduction de la Muséofiche sous forme de réseau MPM, 2009-2010.

(c) Stéphanie Kuhn-Likes.

29 Au-delà de la vocation des études de programmation à traduire une commande d'un maître d'ouvrage à des maîtres d'œuvre, les études de faisabilité doivent s'appuyer sur des études techniques plus complètes sur le site et sur une connaissance totale des collections, afin d'éviter les mauvaises surprises et de préparer au mieux le travail des équipes de maîtrise d'œuvre. L'étude de programmation est ainsi confirmée dans sa vocation pré-opérationnelle permettant de mettre en cohérence des fondements scientifiques et culturels avec des problématiques purement techniques. Ceci a des conséquences autant sur le temps dédié aux études de programmation que sur les budgets à y consacrer. En effet, pour obtenir les résultats souhaités, les études de programmation doivent pouvoir conforter des compétences techniques supplémentaires souvent sousévaluées par les maîtres d'ouvrage (Experts climaticien, BET, nombre de journées suffisantes y compris pour les experts en conservation préventive...). 
Figure 6

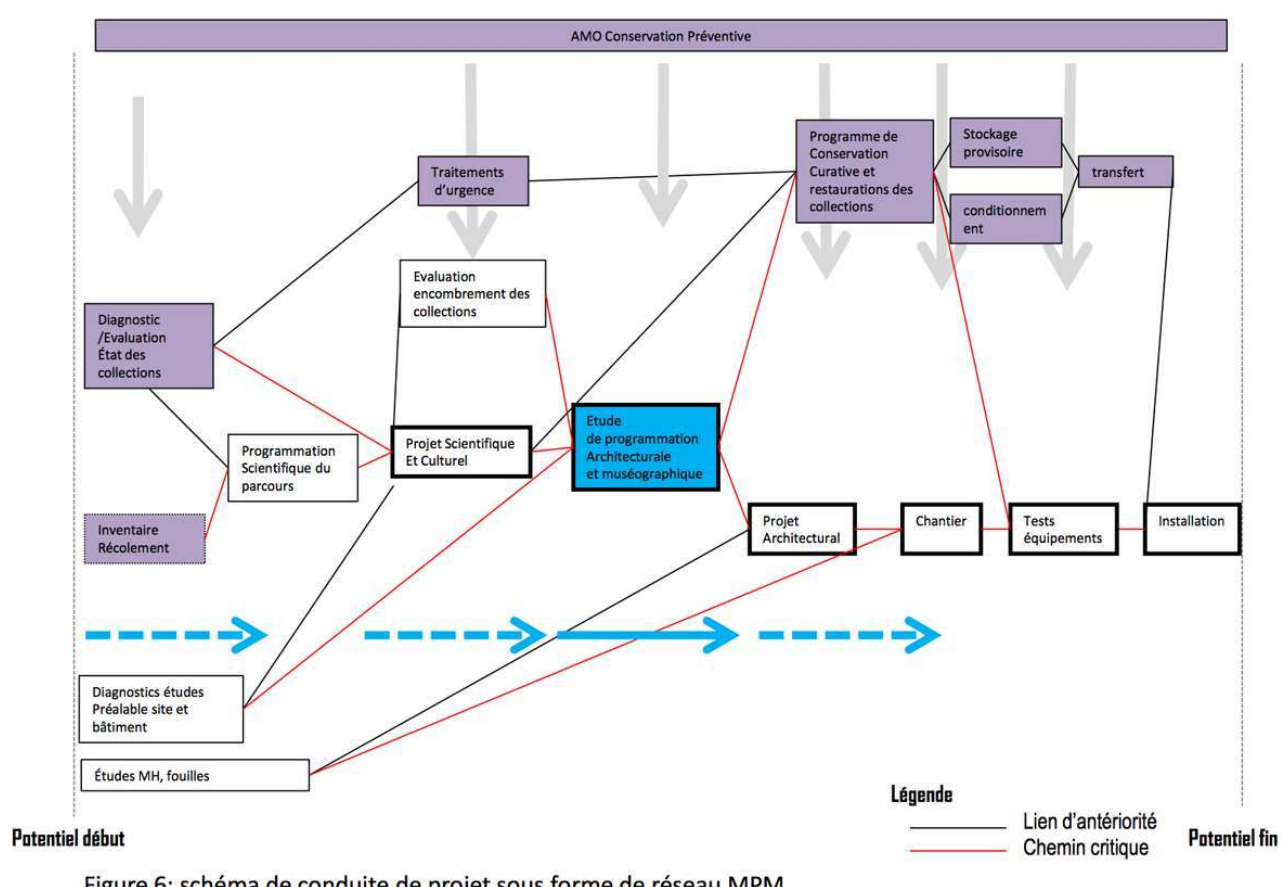

Schéma de conduite de projet sous forme de réseau MPM, 2009-2010.

(c) Stéphanie Kuhn-Likes.

\section{Les ressources capitalisables ou intégrables aux études de programmation dans le cadre d'une optimisation du processus}


sondages/études techniques spécifiques, affinement/recadrage du parcours scientifique et de la sélection en interface avec la programmation muséographique et architecturale.

Dans certains cas, la rédaction du PSC concomitamment aux études de programmation apparaît pertinente à plusieurs égards (gestion optimisée des délais, approche ciblée, mise en perspective avec le site,...). Ceci est envisageable dans la mesure où les collections sont maitrisées par les responsables scientifiques. Les tâches liées à la connaissance des collections peuvent s'organiser aux différentes étapes du projet pour alimenter la production des équipes de programmation puis des équipes de maîtrise d'Cuvre. La connaissance des collections n'est pas considérée comme une analyse indépendante du projet, mais au service de ce dernier. Cette hypothèse de travail nécessite une mobilisation importante des équipes du musée pendant la phase de programmation.

\section{Vers une mise en perspective du rôle des acteurs}

À la question quels moyens mettre en œuvre et quel rôle faire jouer aux acteurs, nous identifions plusieurs perspectives. Ainsi, si la définition des objectifs qualitatifs en faveur de la conservation préventive au service d'un projet muséographique de médiation global reste majeure, il y a plusieurs façons de s'appuyer sur les différents acteurs mobilisables en interne ou en externe, publics ou privés.

\section{Prestataires privés}

- Considérer les critères de conservation des collections comme une donnée fondamentale du programme impactant la fonctionnalité, les orientations architecturales et techniques et les budgets ;

- Généraliser les missions $\mathrm{AMO}^{11}$ prises en charge par les équipes de programmation intégrant des compétences en conservation préventive pour coordonner l'ensemble des actions à mener et la cohérence du projet global (scientifique, culturel, technique) a minima jusqu'aux phases PRO et DCE ;

- Intégrer systématiquement dans les équipes de programmations des préventistes associés aux autres expertises habituelles ;

- Renforcer les échanges interdisciplinaires (architectes, scénographes, conservateurs, préventistes, programmistes, BET, ...) sur les problématiques propres aux musées.

\section{Acteurs publics}

- Améliorer les moyens mis en œuvre par l'État auprès des Musées de France et notamment des petites collectivités pour apporter son rôle d'expertise et de conseil ;

- Mettre à disposition des ressources et des conseils clairs auprès des équipes de programmistes et des architectes/muséographes ;

- Développer la communication auprès des maîtres d'ouvrage sur les impacts positifs sur le long terme des choix privilégiant la conservation préventive dès les phases d'études. Ceci ayant des incidences sur le budget des missions de programmation ;

- Capitaliser au maximum les informations sur les projets en cours et déjà réalisés pour bénéficier des retours d'expériences : mise en place d'une sorte de Base de Données-veille. 


\section{NOTES}

1. - Voir le site : Master Conservation-Restauration des biens culturels.

2. - Voir la Loi du 4 janvier 2002 relative aux musées de France codifiée au Livre IV du code du patrimoine.

3. - KUHN-LIKES, Stéphanie. L'intégration de la Conservation Préventive dans les projets de création et de rénovation de musées, de la genèse à la première année d'exploitation, Problématique de l'intégration de la Conservation Préventive dans la conduite de projet. Mémoire de Master II, Conservation des biens culturels, Université Paris I - année 2009-2010.

4. - Voir le site : www.archi.fr/MIQCP/IMG/pdf/MEDIATIONS_18.pdf.

5. - Voir l'extrait de la fiche médiation : www.archi.fr/MIQCP/IMG/pdf/MEDIATIONS_18.pdf.

6. - Nous pouvons citer quelques exemples récents : Louvre-Lens, le musée du Quai Branly, une partie des salles du musée des Beaux-Arts d'Angoulême...

7. - BET : Bureaux d'Études Techniques.

8. - Muséofiche : Schéma d'élaboration d'un projet de musée, source SMF (voir figures 4 et 5).

9. - Il ne s'agit pas de remettre en cause le PSC comme document structurant pour un musée. Ce type de document est nécessaire à toute institution pour structurer ses objectifs et planifier ses actions, et ce, indépendamment d'un projet architectural et muséographique.

10. - « Ni le programmiste, ni le maître d'œuvre ne peuvent bien travailler sans un document de cadrage initial suffisamment détaillé » commentaire de Marie-Hélène Joly dans l'article «Le Projet Scientifique et Culturel a-t-il de l'avenir? ». Dans La lettre de l'Ocim, n¹24, juillet-août 2009, p. 11.

11. - Assistance à maîtrise d'ouvrage.

\section{RÉSUMÉS}

Malgré les progrès réalisés depuis une dizaine d'années, la conservation préventive reste encore majoritairement pensée comme une discipline liée aux collections stricto sensu, notamment par les hommes de l'art, les programmistes, et différents prestataires intervenant sur un projet. Si les études de conservation préventive se sont largement développées, celles-ci influent-elles suffisamment sur les projets architecturaux et muséographiques ? Comment cette discipline estelle intégrée dans les phases clés et notamment dans la phase de programmation architecturale et muséographique? Quels sont les enjeux qui sous-tendent une meilleure intégration de la conservation préventive dans les différentes phases de réalisation d'un projet de création ou de refonte d'un musée ? Au-delà de la phase de programmation qui constitue un moment charnière dans le processus d'un projet de création ou de rénovation de musée, l'enjeu du travail réalisé dans le cadre du mémoire de Master de Conservation Préventive de l'université de Paris I, était de problématiser l'intégration de la conservation préventive dans tout le processus de conduite de projets, du PSC jusqu'à la livraison des équipements. Il s'agissait également d'identifier de façon objective les indicateurs-clés pouvant considérablement influencer le fonctionnement 
futur des institutions muséales, de repérer les schémas actuels d'intégration de la conservation préventive et de ses spécialistes au cours de la réalisation des projets. La démarche proposée s'inscrit dans le cadre d'une évaluation de gestion de projet.

In spite of all the progress achieved over the past ten years, preventive conservation is still largely considered as a discipline related to the collections strictly speaking, in particular by architects, space planers and other stakeholders involved on a project. Although investigations on preventive conservation have been largely developed, do they have enough effect on architectural and museum projects? How is this discipline integrated within the key phases and especially within the one of architectural and museum planning? What are the stakes of the better preventive conservation integration within different phases of a project to create or redesign a museum? Beyond the planning phase which is a key moment in the process of structuring a project on a museum creation or renovation, what is at stake in the work achieved within the Preventive Conservation Master's thesis, is to develop problematics about the integration of preventive conservation in the whole process of project management, from the PSC to the equipment delivery, and to objectively identify the key indicators that can have, in the future, a significant influence upon museum institutions operating model, and at last to identify during the project execution the current outline of the integration of both the preventive conservation and its experts. The proposed approach comes within the scope of a project management appraisal.

\section{AUTEUR}

\section{STÉPHANIE KUHN}

Responsable d'étude-gérant, ASK stephaniekuhn-ask@bbox.fr 